Afatinib for an elderly NSCLC patient with chronic kidney disease

Short running title: Afatinib for a patient with CKD

Shinichiro Okauchi, Satoshi Hagimoto, Hajime Osawa, Gen Ohara, Hiroaki Satoh

Division of Respiratory Medicine, Mito Medical Center, University of Tsukuba-Mito Kyodo General Hospital

Correspondence: Hiroaki Satoh, MD

Division of Respiratory Medicine, Mito Medical Center, University of Tsukuba, Miya-machi 3-2-7, Mito, Ibaraki, 310-0015, Japan

Tel: +81-29-231-2371, Fax: +81-29-221-5137,

E-mail: hirosato@md.tsukuba.ac.jp

Keywords: afatinib; lung cancer; chronic kidney disease 
Afatinib, a second-generation epidermal growth factor receptor-tyrosine kinase inhibitor (EGFR-TKI), prolonged survival in EGFR-mutated non-small cell lung cancer (NSCLC) patients [1]. Due to the increase in the incidence of lung cancer among elderly individuals, the efficacy and safety of EGFR-TKIs for the treatment of elderly patients with non-small-cell lung cancer (NSCLC) have become interested. Aging is associated with a higher prevalence of comorbid disease. Most patients at the time of diagnosis of NSCLC have suffered some types of comorbidities such as chronic kidney disease (CKD), contraindicating any chemotherapy. Afatinib has some dosage forms so that it can be reduced, and afatinib and its metabolites are not excreted predominantly via the urine [2]. Successful afatinib treatment of advanced NSCLC patients undergoing hemodialysis have already reported [3, 4]. In this report, we present a successfully treatment of an advanced NSCLC patient, who was unsuitable for chemotherapy because of CKD, start with afatinib at the dose of $20 \mathrm{mg} /$ day.

A 79-year-old woman, never smoker, was diagnosed with stage IIIB lung adenocarcinoma. Eastern Cooperative Oncology Group (ECOG) performance status (PS) was 1. She underwent a right superior lung lobectomy plus mediastinal lymph nodes dissection. An EGFR exon 21 L858R was 
identified in the resected tumor. Six months after surgery, she developed pulmonary metastases. In the meanwhile, she had CKD (serum creatinine level $1.18 \mathrm{mg} / \mathrm{dl}$, blood urea nitrogen level $28 \mathrm{mg} / \mathrm{dl}$, and creatinine clearance (CC) 34 $\mathrm{ml} / \mathrm{min}$ according to the cockroft and gault formula. She started afatinib (20mg a day). Renal function was checked twice monthly, with CC ranging between 32 and $48 \mathrm{ml} / \mathrm{min}$ (serum creatinine levels between 0.86 and $1.43 \mathrm{mg} / \mathrm{dl}$ ). Nineteen months after the initiation of afatinib therapy, the patient is still well on treatment, achieving partial response.

In our patient, the choice of EGFR-TKI was based on the need for palliative treatment. Afatinib has tablets that can be reduced in several stages. The starting dose of afatinib was $20 \mathrm{mg}$ a day, supposing that if side effects appeared it would be further reduced. However, further renal function was not impaired by afatinib therapy, and no other severe toxicity was developed. Afatinib and its metabolites are excreted predominantly via the feces, with urinary excretion representing a minor elimination pathway (4.29\%)[2]. To the best of our knowledge, this is the first report in which erlotinib was administered in elderly patients with CKD achieving disease control. EGFR-TKI is the cornerstone for the treatment of advanced EGFR-mutated 
NSCLC patients. However, its utility on patients with CKD has largely been unstudied, because most cancer drug trials exclude such patients. Afatinib can become one treatment option for patients with NSCLC and CKD.

\section{Disclosure of interest}

The authors declare that there is no conflicts of interest concerning this article. 
References

1. Keating GM. Afatinib: A Review in Advanced Non-Small

Cell Lung Cancer. Target Oncol 2016; 11: 825-35.

2. Stopfer $\mathrm{P}$, Marzin $\mathrm{K}$, Narjes $\mathrm{H}$, et al. Afatinib

pharmacokinetics and metabolism after oral administration

to healthy male volunteers. Cancer Chemother Pharmacol

$2012 ; 69: 1051-61$.

3. Bersanelli M, Tiseo M, Artioli F, Lucchi L, Ardizzoni A. Gefitinib and afatinib treatment in an advanced non-small cell lung cancer (NSCLC) patient undergoing hemodialysis. Anticancer Res 2014; 34: 3185-8.

4. Imai H, Kaira K, Naruse I, et al. Successful afatinib treatment of advanced non-small-cell lung cancer patients undergoing hemodialysis. Cancer Chemother Pharmacol 2017; 79: $209-13$. 